ÊESTI NSV TEADUSTE AKADEEMIA TOIMETISËD̂.

FUOSIKA * MATEMAATIKA

ИЗВЕСТИЯ АКАДЕМИИ НАУК ЭСТОНСКОИ ССР. ФИЗИКА * МАТЕМАТИКА

PROCEEDINGS OF THE ACADEMY OF SCIENCES OF THE ESTONIAN SSR. PHYSICS * MATHEMATICS

$1989,38,2$

\title{
ПРИЧИННЫЕ РЕШЕТКИ ДИФРАКЦИИ В ПРОСТРАНСТВЕННО- ВРЕМЕННОЙ ГОЛОГРАФИИ
}

\author{
(Представил П. Саари)
}

B $\left[{ }^{1-3}\right]$ предложен и экспериментально реализован обобщенный метод голографии, позволяющий записывать и восстанавливать не только пространственные, но и временные характеристики импульсного светового поля. Достигается это благодаря использованию фотохромных сред спектрально высокоселективной светочувствительности. Примером таковых является низкотемпературная твердая матрица с фотовыжигаемыми примесными молекулами, обладающая широким неоднородно уширенным спектром поглощения, образованным из узких бесфононных линий примесных молекул [ $\left.{ }^{4}\right]$.

Теория, описывающая получение и считывание таких 4-мерных $\mathbf{r}, t$-голограмм изложена в $\left[{ }^{5,6}\right]$. Следует подчернуть, что $\mathbf{r}, t$-голограмма, записанная в высокоселективно фотохромной среде, по некоторым свойствам существенно отличается от обыкновенной. Во-первых, при $\mathbf{r}, t$-голографии в среде формируется не одна, а огромное число дифракционных решеток - одна решетка для каждого монохроматического компонента падающего света *. При считывании такой голограммы существенную роль играет принцип причинности, который накладывает специальные ограничения на возникновение некоторых дифракционных порядков [ $\left.{ }^{5}\right]$. В свете сказанного целесообразно введение термина «причинные решетки» для составляющих $\mathbf{r}, t$-голограмму пространственно-спектральных периодических структур в диэлектрической проницаемости среды. Кроме того, при записи $\mathbf{r}, t$-голограммы значительными могут оказаться эффекты насыщения и нелинейности фоточувствительности, которые, в свою очередь, ведут к появлению дополнительных эффектов, например, к возникновению высших порядков дифракции в восстановительном поле [ $\left.{ }^{6}\right]$.

Вышеотмеченные и многие другие свойства делают весьма интересным и необходимым теоретическое исследование причинных решеток, что и является задачей данной работы. Практической целью исследования является обобщение развитой в $\left[{ }^{5,6}\right]$ теории на случай объемной (толстой) голограммы, которая наиболее адекватно соответствует экспериментальной ситуации.

В первой части работы модифицируется метод «связанных волн», хорошо известный в традиционной голографии $\left[{ }^{7,8}\right]$, для исследования дифракции света в причинных решетках. Во второй части обсуж-

* Спектральная ширина индивидуального компонента определяется полушириной линии поглощения одной примесной молекулы и составляет в типичных случаях $10^{6}-10^{9}$ Гц. 
даются́ полученные резул̄ьтаты и выводяิтся оценки дифракционной эффективности (ДЭ) для нескольких первых порядков рассеяния, которые являются достаточно интенсивными для экспериментального наблюдения $\left[{ }^{9}\right]$.

\section{1. Теория дифракции в причинных решетках}

Распространение каждого монохроматического компонента $E(\mathbf{r}, \omega)$ поля светового импульса $E(\mathbf{r}, t)$ в пространственно-спектрально неоднородной среде пластинки $\mathbf{r}, t$-голограммы описывается волновым уравнением **

$$
\nabla^{2} E(\mathbf{r}, \omega)+\frac{\omega^{2}}{c^{2}} \varepsilon(\mathbf{r}, \omega) E(\mathbf{r}, \omega)=0
$$

где $c$ - скорость света в вакууме. При решении (1) существенную роль играет вид комплексной диэлектрической проницаемости среды $\varepsilon(\mathbf{r}, \omega)$. Последнюю определяем, рассматривая возбужденные светом примесные молекулы, как классические гармонические осцилляторы, описываемые уравнением движения

$$
\frac{\partial^{2}}{\partial t^{2}} R_{i}(\mathbf{r}, t)+2 \Gamma \frac{\partial}{\partial t} R_{i}(\mathbf{r}, t)+\omega_{i}^{2} R_{i}(\mathbf{r}, t)=\frac{e}{m} E(\mathbf{r}, t),
$$

где $R_{i}(\mathrm{r}, t)$ - амплитуда колебания осциллятора с собственной частотой $\omega_{i}$, расположенного в точке пластинки с радиус-вектором $\mathbf{r} ; \mathbf{\Gamma}, e$ и $m$ соответственно затухание, заряд и эффективная масса, предполагаемые одинаковыми для всех осцилляторов. Решая (2) преобразованием Фурье, находим:

$$
R_{i}(\mathbf{r}, \omega)=\frac{\frac{e}{m} E(\mathbf{r}, \omega)}{\omega_{i}^{2}-\omega^{2}+2 i \Gamma \omega}
$$

Далее, считая, что распределение осцилляторов по собственным частотам $\omega_{i}$ и пространственным координатам $\mathbf{r}$ описывается непрерывной функцией $g\left(\mathbf{r}, \omega_{i}\right)$, и производя суммирование по амплитудам (3), можем для $\varepsilon(\mathbf{r}, \omega)$ написать:

$$
\varepsilon(\mathbf{r}, \omega)=\varepsilon_{0}+\frac{4 \pi e^{2}}{m} \int_{-\infty}^{\infty} \frac{g\left(\mathbf{r}, \omega_{i}\right) d \omega_{i}}{\omega_{i}^{2}-\omega^{2}+2 i \Gamma \omega} .
$$

Через $\varepsilon_{0}$ здесь обозначена средняя диэлектрическая проницаемость среды, не обусловленная рассматриваемыми (бесфононными) переходами. В случае $\mathbf{r}, t$-голограммы функция $g\left(\mathbf{r}, \omega_{i}\right)$ соответствует некоторому конкретному распределению, полученному для данного сигнального поля в данных условиях экспозиции. Естественно предполагать, что вероятность фотопревращения индивидуальной молекулы в ходе экспозиции пропорциональна поглощенной ей полной энергии. В таком случае $g\left(\mathbf{r}, \omega_{i}\right)$ выражается в виде экспонента

$$
g\left(\mathbf{r}, \omega_{i}\right)=g_{0} \exp \left[-t_{E} \chi \int_{-\infty}^{\infty} \frac{\Gamma}{\pi} \frac{I(\mathbf{r}, \omega) d \omega}{\left(\omega_{i}-\omega\right)^{2}+\Gamma^{2}}\right],
$$

\footnotetext{
** Предположено, что диэлектрические свойства пластинки голограммы не изменяются в направлении электрического вектора внешнего поля. При этом в данной работе не учитываются эффекты, связанные с поляризацией поля.
} 
где $g_{0}-$ распределение осцилляторов до экспозиции, предполагаемое здесь постоянным, $t_{E}$ - время экспозиции, $x-$ коэффициент выжигания, $I(\mathbf{r}, \omega)$ - спектральная интенсивность экспонирующего света. При получении (5) учтено условие резонанса и слабого поглощения

$$
\begin{aligned}
& \omega_{i}, \omega \gg\left|\omega_{i}-\omega\right|, \\
& \omega_{i}, \omega \gg \Gamma .
\end{aligned}
$$

Справедливость формулы (5) подтверждается рядом работ, как экспериментальных $\left[{ }^{10,11}\right]$, так и теоретических $\left[{ }^{11,12}\right]$.

При подстановке (5) и (4) в (1) получается интегродифференциальное уравнение, которое в общем случае не имеет аналитического решения. Для упрощения вычислений в настоящей работе ограничимся случаем пространственной и спектральной периодичности функции $I(\mathbf{r}, \omega)^{* * *}$. Соответствующая ситуация реализуется, например, при выжигании голограммы двумя короткими $\delta$-импульсами (см. рис. 1)

$$
E_{\text {экс. }}(\mathbf{r}, t)=R_{0}\left[\delta\left(t-\frac{\mathbf{n}_{1} \mathbf{r}}{c}\right)+\delta\left(t-t_{R}-\frac{\mathbf{n}_{2} \mathbf{r}}{c}\right)\right] .
$$

Здесь $R_{0}$ - амплитуда, $t_{R}$ - задержка между импульсами, $\mathbf{n}_{1}$ и $\mathbf{n}_{2}$ единичные векторы в направлении распространения соответствующих импульсов. Для спектральной интенсивности света (7) найдем:

$$
I(\mathbf{r}, \omega)=2 R_{0}^{2}(1+\cos M \omega),
$$

где $M=t_{R}+\frac{\left(\mathrm{n}_{2}-\mathrm{n}_{1}\right) \mathrm{r}}{c}$.

Теперь интеграл в (5) легко вычисляется

$$
\frac{\Gamma}{\pi} \int_{-\infty}^{\infty} \frac{I(\mathbf{r}, \omega) d \omega}{\left(\omega_{i}-\omega\right)^{2}+\Gamma^{2}}=2 R_{0}^{2}\left\{1+\exp \left[-\Gamma\left|t_{R}\right|\right] \cos M_{\omega_{i}}\right\},
$$

где предположено, что импульсы не действуют на пластинку одновременно, т. е. в каждой точке пластинки выполнено условие

$$
\left|t_{R}\right| \gg\left|\frac{\left(\mathbf{n}_{2}-\mathbf{n}_{1}\right) \mathbf{r}}{c}\right| \text {. }
$$

При этом в экспоненте (9) $|M|$ заменено на $\left|t_{R}\right|$. Поскольку, согласно (9) и (5),$g\left(\mathbf{r}, \omega_{i}\right)$ является периодической функцией аргумента $\omega_{i} \mathrm{c}$ периодом $2 \pi / M$, то его можно разложить в ряд Фурье

$$
g\left(\mathbf{r}, \omega_{i}\right)=g_{0} \sum_{n=-\infty}^{\infty} C_{n} e^{i n \omega_{t} M}
$$

где

$$
C_{n}=e^{-B}(-1)^{n} I_{n}\left(B^{\prime}\right) .
$$

Здесь $I^{n}(x)$ - модифицированная функция Бесселя $n$-го порядка мнимого аргумента, $B=2 R_{0}^{2} x t_{E}-$ средняя экспозиция одиночного мгновенного импульса, а $B^{\prime}=2 R_{0}^{2} x t_{E} \exp \left[-\Gamma\left|t_{R}\right|\right]-$ эффективная экспозиция, обусловленная интерференционными членами двух разделенных во времени импульсов. Как видно, вследствие релаксации в среде $B^{\prime}$ всегда меньше $B$. Только при низких температурах $(\Gamma \rightarrow 0)$ при достаточно короткой $t_{R} B \sim B^{\prime}$.

*** В этом частном случае качественно сохраняются общие свойства причинных решеток. 


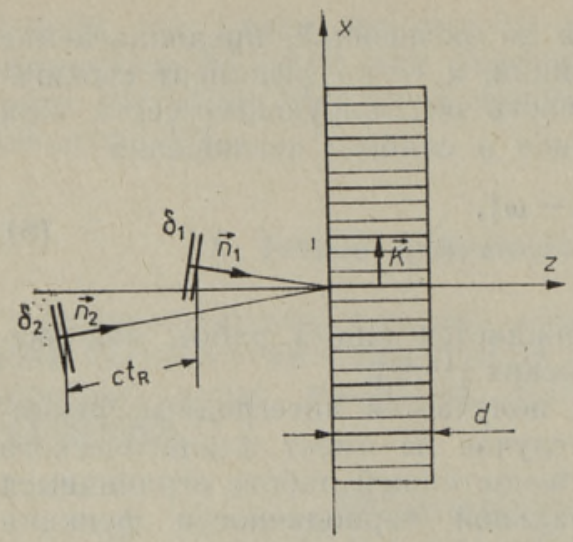

Рис. 1. Запись $\mathbf{r}, t$-голограммы с двумя короткими импульсами $\delta_{1}$ и $\delta_{2} ; d-$ толщина пластинки, $t_{R}-$ задержка между импульсами, K - вектор пространственной решетки

$$
\left(\mathbf{K}=\frac{\omega\left(\mathbf{n}_{2}-\mathbf{n}_{1}\right)}{c}\right) \text {. }
$$

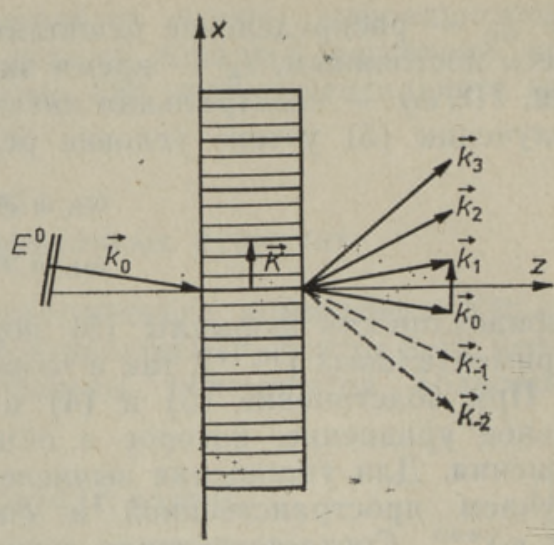

Рис. 2. Восстановление $\mathbf{r}, t$-голограммы; $E^{0}$ - считывающий импульс, ... $\mathbf{k}_{-1}, \mathbf{k}_{0}$, $\mathbf{k}_{1} \ldots$ - волновые векторы светового импульса соответствующего порядка дифракций. Согласно теории дифракционные порядки с волновыми векторами $\mathbf{k}-1$, $\mathbf{k}_{-2} \ldots .$. обозначенные на рисунке прерывистыми линиями, возбуждаться не могут.

Подставляя (11) в (4) и вычисляя интеграл (с учетом (6) и (10)), находим

$$
\begin{gathered}
\varepsilon(\mathbf{r}, \omega)=\varepsilon_{0}-\frac{4 \pi^{2} e^{2} g_{0} i e^{-B}}{m_{\omega}} \times \\
\times\left\{I_{0}\left(B^{\prime}\right)+2 \sum_{n=1}^{\infty}(-1)^{n} I_{n}\left(B^{\prime}\right) \exp \left[-n\left(\Gamma\left|t_{R}\right|+i \omega M\right)\right]\right\} .
\end{gathered}
$$

В силу (10) знак величины $M$ определяется знаком задержки $t_{R}$. Пусть $t_{R}>0$, т. е. импульс с направления $\mathrm{n}_{1}$ подавался на пластинки раньше, чем импульс с направления $\mathbf{n}_{2}$. При этом (12) можно переписать в виде:

$$
\begin{gathered}
\varepsilon(\mathbf{r}, \omega)=\varepsilon_{0}-\frac{i T e^{-B} c \sqrt{\varepsilon_{0}}}{\omega} \times \\
X\left\{I_{0}\left(B^{\prime}\right)+2 \sum_{n=1}^{\infty}(-1)^{n} I_{n}\left(B^{\prime}\right) \exp \left[-n\left(i \omega t_{R}+i \mathbf{K r}+\Gamma t_{R}\right)\right]\right\},
\end{gathered}
$$

где $T=\frac{4 \pi^{2} e^{2} g_{0}}{m c \sqrt{\varepsilon_{0}}} \quad-$ коэффициент затухания в неэкспонированной пластинке и $\mathbf{K}=\frac{\left(\mathbf{n}_{2}-\mathbf{n}_{1}\right) \omega}{c}-$ вектор пространственной решетки. Если $t_{R}<0$, то полученная формула существенно не изменяется: тогда в (13) следует $t_{R}$ заменить на $\left|t_{R}\right|$ и $\mathbf{K}$ на $-\mathbf{K}$.

Для решения волнового уравнения (1) используем метод связанных волн (подробнее см. $\left[{ }^{8,13,14}\right]$ ). При этом предполагаем, что световое поле $E(\mathbf{r}, \omega)$ внутри пластинки состоит из суммы световых полей всевозможных порядков дифракции

$$
E(r, \omega)=\sum_{l=-\infty}^{\infty} E_{l}(z, \omega) \exp \left[-i \mathbf{k}_{l} \mathbf{r}\right]
$$

где $E_{l}(z, \omega)$ и $\mathbf{k}_{l}$ соответственно амплитуда и волновой вектор $l$-го порядка дифракции. Для удовлетворения закона сохранения квазиим- 
пульса (условиям Брегга) при отражении света на неоднородностях среды векторы $\mathbf{k}_{l}$ в (14) должны быть связанными между собой

$$
\mathrm{k}_{l}=\mathrm{k}_{0}+l \mathbf{K}, \quad l=0, \pm 1, \pm 2, \pm 3 \ldots .
$$

Здесь $\mathbf{k}_{0}$ соответствует недифрагированному свету, т. е. характеризует направление считывания. Его абсолютное значение определено как среднее волновое число плоской монохроматической волны в пластинке при отсутствии бесфононного поглощения: $\left|\mathrm{k}_{0}\right|=\frac{\omega}{c} \sqrt{\varepsilon_{0}}$. Взаимное расположение векторов $\mathbf{k}_{0}, \mathbf{k}_{l}$ и $\mathbf{K}$ показано на рис. 2. Подставляя (14) и (13) в (1) и пренебрегая при этом в соответствии с теорией Когельника $\left[{ }^{8}\right]$ вторыми производными вида $\frac{\partial^{2}}{\partial z^{2}} E_{l}(z, \omega)$ и приравнивая с учетом (15) нулю коэффициенты при разных экспонентах $\exp \left(i \mathbf{k}_{l} \mathbf{r}\right)$, получим для определения амплитуд $E_{l}(z, \omega)$ бесконечную систему дифференциальных уравнений первого порядка

$$
\begin{gathered}
2 k_{l z} \frac{\partial}{\partial z} E_{l}(z, \omega)- \\
-\left[i\left(\left|\mathrm{k}_{l}\right|^{2}-\left|\mathrm{k}_{0}\right|^{2}\right)-T e^{-B}\left|\mathrm{k}_{0}\right| I_{0}\left(B^{\prime}\right)\right] E_{l}(z, \omega)+2 T e^{-B}\left|\mathbf{k}_{0}\right| \times \\
\times \sum_{n=1}^{\infty}(-1)^{n} I_{n}\left(B^{\prime}\right) \exp \left[-n\left(i \omega t_{R}+\Gamma t_{R}\right)\right] E_{l-n}(z, \omega)=0 .
\end{gathered}
$$

Здесь $k_{l z}-z-$ компонета волнового вектора $\mathbf{k}_{l}$.

\section{2. Общие свойства и оценка ДЭ r,t-голограммы}

Следует отметить, что система кинетических уравнений (16) существенно отличается от аналогичных систем, найденных для вычисления ДЭ обычных толстых голограмм. А именно, в случае обычных голограмм суммирование в кинетических уравнениях типа (16) по разным порядкам дифракции проводится от $-\infty$ до $\infty$ (см. напр. $\left.\left[{ }^{14}\right]\right)$. Это значит, что в таких средах амплитуда каждого данного порядка дифракции определенным образом связана с амплитудами всех других порядков. Следовательно, может иметь место переход энергии светового поля между любыми порядками в любом направлении. Но в нашем случае, поскольку здесь суммирование в кинетических уравнениях (16) проводится только по более низким порядкам, энергия может протекать только в одном направлении - от более низких к более высоким. Характерный для обычной голограммы обратный поток здесь не может иметь место. Такое особое свойство r, $t$-голограмм следует непосредственно из принципа причинности. А именно, поскольку процедура восстановления $\mathbf{r}, t$-голограммы является аналогом процесса формирования сигнала стимулированного фотонного эха, то высшие порядки дифракции могут здесь появляться только в более поздние моменты времени чем низшие.

В кинетических уравнениях (16) это выражается наличием фазовогомножителя $\exp \left(-i n \omega t_{R}\right)$, обеспечивающего временной сдвиг на $n t_{R}$ при обратном фурье-преобразовании спектральной амплитуды $E_{l}(z, \omega)$. Тогда и очевидно, что более поздние импульсы высшего порядка не могут влиять на более ранние импульсы низшего порядка. Отсюда следует одно из основных различий между объемной обычной и объемной $\mathbf{r}, t$-голограммой. А именно, процесс дифракции света в обычной голограмме всегда обратимый в том смысле, что внутри каждой достаточно толстой голограммы всегда найдется такая плоскость, где полностью или, по меньшей мере, почти точно восстанавливается 
первоначальное распределение энергии светового поля между разными порядками дифракции, которое имеет место на передней плоскости голограммы. Сказанное, разумеется, относится к случаю достаточно слабого поглощения в пластинке.

Но в $\mathbf{r}, t$-голограмме с увеличением толщины пройденного светом слоя энергия поля может перейти только в сторону более высших порядков. Здесь первоначальное распределение энергии никогда не восстанавливается.

Обращаясь к системе (16), обнаруживаем, что она аналитически разрешима для амплитуд сколь угодно высокого порядка дифракции. Следует отметить, что при обычных голограммах соответствующие вычисления в общем случае осуществимы только числовыми методами на ЭВМ.

Для решения (16) учтем начальные условия: в передней плоскости голограммы существует только недифрагированная компонента

$$
\left.E_{l}(z, \omega)\right|_{z=0}=\left\{\begin{array}{lll}
E^{0}(\omega), & \text { если } l=0 \\
0, & \text { если } l \neq 0
\end{array}\right.
$$

где $E^{0}(\omega)$ - спектральная амплитуда считывающего света до голограммы.

Рассмотрим произвольный порядок с индексом $l<0$. В силу условий (17) $\left.E_{l}(z, \omega)\right|_{z=0}=0$, а в силу уравнения (16) $\left.\frac{\partial}{\partial z} E_{l}(z, \omega)\right|_{z=0}=0$. Отсюда непосредственно следует, что все $E_{l}(z, \omega)$ при $l<0$ тождественно равны нулю при любом значении аргумента $z$. Следовательно, дифракционные порядки с индексом $l<0$ не могут возбуждаться (см. рис. 2).

Перейдем теперь к оценке ДЭ $\mathbf{r}, t$-голограммы в разные порядки. Для амплитуды недифрагированного компонента, т. е. для порядка $l=0$ из (16) находим

$$
E_{0}(z, \omega)=E^{0}(\omega) \exp \left[-\frac{T e^{-B} I_{0}\left(B^{\prime}\right) z}{2 \cos \varphi}\right]
$$

где $\varphi$ - угол между вектором $\mathbf{k}_{0}$ и осью $z: \cos \varphi=\frac{\left|k_{0 z}\right|}{\left|\mathbf{k}_{0}\right|}$. Понимая ДЭ $\mathbf{r}, t$-голограммы как отношение энергии прошедшего пластинку импульса к энергии падающего (считывающего) импульса для нулевого порядка из (18) находим:

$$
\eta_{0}=\frac{\left.\int\left|E_{0}(z, \omega)\right|^{2}\right|_{z=d} d \omega}{\int\left|E^{0}(\omega)\right|^{2} d \omega}=\exp \left[-\frac{T e^{-B} I_{0}\left(B^{\prime}\right) d}{\cos \varphi}\right],
$$

где $d$ - толщина пластинки. Қак видно, интенсивность нулевого порядка в пластинке экспоненциально уменьшается со скоростью $\frac{T e^{-B} I_{0}\left(B^{\prime}\right)}{\cos \varphi}$.

Полагая в (16) $l=1$ и учитывая (17), для первого порядка дифракции находим

$$
E_{1}(z, \omega)=T e^{-B} \frac{\left|\mathbf{k}_{0}\right|}{\left|k_{1 z}\right|} I_{1}\left(B^{\prime}\right) E^{0}(\omega) C_{1}(z, \omega) \exp \left[-i \omega t_{R}-\Gamma t_{R}+u_{1} z\right] .
$$

Здесь $\quad C_{1}(z, \omega)=\frac{\exp \left[-\left(u_{0}+u_{1}\right) z\right]-1}{-\left(u_{0}+u_{1}\right)}$,

$\mu_{1}=\frac{i\left(\left|k_{1}\right|^{2}-\left|\mathbf{k}_{0}\right|^{2}\right)-T e^{-B}\left|\mathrm{k}_{0}\right| I_{0}\left(B^{\prime}\right)}{2\left|k_{1 z}\right|}$ и $u_{0}=\frac{T e^{-B} I_{0}\left(B^{\prime}\right)\left|\mathbf{k}_{0}\right|}{2\left|k_{0 z}\right|}$. 
Умножая (20) на комплексно-сопряженную величину, находим условия, при которых ДЭ первого порядка достигает своего максимального значения

$$
\begin{aligned}
& \text { 1. }\left|k_{0 z}\right|=\left|k_{1 z}\right| \\
& \text { 2. }\left|\mathbf{k}_{0}\right|=\left|\mathbf{k}_{1}\right|, \\
& \text { 3. } T e^{-B}\left|\mathbf{k}_{0}\right| d=\frac{2\left|k_{0 z}\right|}{I_{0}\left(B^{\prime}\right)} .
\end{aligned}
$$

Первые два равенства в (22) связаны с геометрической схемой записи и восстановления голограммы. Они выражают, соответственно, известные уже в традиционной голографии правила (см. $\left.\left[{ }^{7,8}\right]\right)$ : ДЭ первого порядка является максимальной, если, во-первых, вектор пространственной решетки $\mathbf{K}$ перпендикулярен оси $z$ (при этом, согласно (15), $\left.\left|k_{0 z}\right|=\left|k_{1 z}\right|\right)$, во-вторых, считывание голограммы проводится под углом Брегга (при этом $\left.\left|\mathbf{k}_{0}\right|=\left|\mathbf{k}_{1}\right|\right)$. Если последнее условие не выполнено, то согласно $(21)$, коэффициент $C_{1}(z, \omega)$ зависит и от частоты $\omega$ (поскольку волновые векторы $\mathbf{k}_{1}$ и $\mathbf{k}_{0}$ зависят от него). При этом ДЭ для разных монохроматических компонентов считывающего света является разной, что ведет к возникновению существенных искажений при восстановлении $\mathbf{r}, t$-голограммы.

Третье условие в (22) выражает оптимальное соотношение между первоначальным коэффициентом поглощения пластинки $T d$ и экспозицией $B$.

Если (22) выполнено, то для ДЭ первого порядка находим

$$
\eta_{\mathrm{I}}=4 e^{-2} e^{-2 \Gamma t_{R}} \cdot\left(\frac{I_{1}\left(B^{\prime}\right)}{I_{0}\left(B^{\prime}\right)}\right)^{2} .
$$

В действительности всегда $I_{1}\left(B^{\prime}\right)<I_{0}\left(B^{\prime}\right)$, только при $B^{\prime} \rightarrow \infty$ $\frac{I_{1}\left(B^{\prime}\right)}{I_{0}\left(B^{\prime}\right)} \rightarrow 1$. В таком случае (считая $\Gamma \rightarrow 0$ ) находим: $\eta_{\mathrm{I}}=4 e^{-2} \approx 54 \%$.
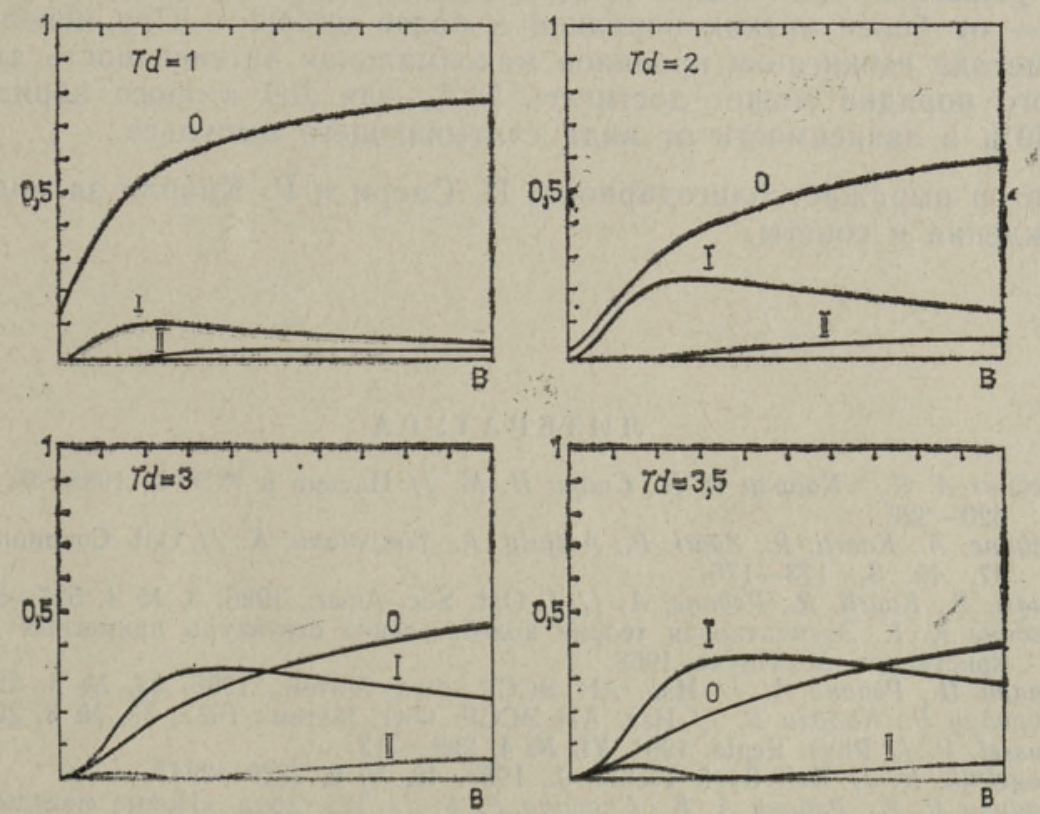

Рис. 3. Дифракционная эффективность $\mathbf{r}$, $t$-голограммы нулевого (0), первого (I) и второго (II) порядка в зависимости от экспозиции $B$ при разных оптических толщинах $T d$ пластинки. 
Однако практически такое высокое значение ДЭ не достигаемо, поскольку при его теоретическом выводе мы пренебрегли:

1) наличием релаксации в среде: $\Gamma \neq 0$;

2) наличием других механизмов поглощения в среде, кроме бесфононных переходов;

3) ослаблением света в среде при описании процесса записи голограммы.

Кроме того, условие $B^{\prime} \rightarrow \infty$ требует, согласно (22), использования пластинки с бесконечно большим коэффициентом поглощения $T d$, поскольку $\lim _{B \rightarrow \infty} e^{-B} I_{0}(B)=0$. Практически это не достигаемо и противоречит пункту 3 в (22).

Учитывая (18), находим, что при условии (22), когда интенсивность первого порядка является максимальной $(\sim 54 \%)$, интенсивность прямораспространяющегося импульса $(\sim 13 \%)$ может оказаться значительно слабее. Такое явление неоднократно наблюдалось и в эксперименте $\left[{ }^{5}\right]$.

Вычисление ДЭ второго и более высоких порядков ведет к трудоемким и сложным аналитическим выражениям. Кроме того, в случае высших порядков при $l>1$ нельзя удовлетворять условиям Брегга (22). Следовательно, восстановление высших порядков всегда сопровождается значительными искажениями записанного импульса. В таком случае для вычисления ДЭ целесообразно использовать ЭВМ, а результаты представлять графически (см., напр., рис. 3).

\section{3. Заключение}

Итак, приходим к следующим выводам. В процессе дифракции света на пространственно-спектральных решетках существенную роль играет принцип причинности, который определяет односторонний характер процесса дифракции: в пространственно-спектральных периодических решетках свет может дифрагировать только в одном направлении - от более низких порядков к более высоким. При использовании метода выжигания провалов максимальная интенсивность для ДЭ первого порядка может достигать $54 \%$, для ДЭ второго порядка $30-40 \%$ в зависимости от вида считывающего импульса.

Автор выражает благодарность П. Саари и Р. Каарли за полезные обсуждения и советы.

\section{ЛИТЕРАТУРА}

1. Ребане А. К., Каарли Р. К., Саари П. М. // Письма в ЖЭТФ, 1983, 38, вып. 7, $320-323$,

2. Rebane, A., Kaarli, R., Saari, P., Anijalg. A., Timpmann, K. // Opt. Common., 1983, 47, № 3, $173-176$.

3. Saari, P., Kaarli, R., Rebane, A. // J. Opt. Soc. Amer., 1986, 3, № 4, 527-534.

4. Ребане K. K. Элементарная теория колебательной структуры примесных центров крнсталлов. М., Наука, 1968.

5. Саари П., Ребане А. // Изв. АН ЭССР. Физ. Матем., 1985, 33, № 3, 322-333.

6. Сарапуy $P$., Каарли Р. // Изв. АН ЭССР. Физ. Матем., 1987, 36, № 3, 299-309.

7. Russel, P. // Phys. Repts, 1981, 71, № 4, 209-312.

8. Kogelnik, H. // Bell Syst. Techn., J., 1969, 48, № 9, 2909-2947.

9. Каарли Р. К., Ребане А. К., Сарапуу Р. В. // Тез. докл. «Новые методы лазерной спектроскопии молекул в низкотемпературных средах», Таллин, 1987, $41-42$.

10. Гороховский А. А., Каарли Р. К., Ребане Л. А. // Письма в ЖЭТФ, 1974, 20, вып. 7, 474-479. 
11. Rebane, L., Gorokhovskii, A., Kikas, J. // Appl. Phys., 1982, B29, № 4, 235-250.

12. Кикас Я. В. Некоторые вопросы исследования электронно-колебательных спектров примесных молекул в неоднородных твердотельных матрицах. Автореф. канд. дис. Тарту, 1978.

13. Ярвив А., Юх П. Оптические волны в кристаллах. М.. Мир. 1987.

14. Magnusson, R., Gaylord, T. // J. Opt. Soc. Amer., 1977, 67, № 9, 1165-1170.

Ннститут физики

Академии наук Эстонской ССР
Поступила в редакцию $17 /$ VI 1988

\section{R. SARAPUU}

\section{POOHJUSLIKUD DIFRAKTSIOONIVÕRED AEGRUUMILISES HOLOGRAAFIAS}

On näidatud, et valguse difraktsiooniprotsessi kirjeldamisel ruumiliselt ja spektraalselt perioodilistes keskkondades on olulise tähtsusega pōhjuslikkuse printsiip. Vastavalt sellele on üheselt määratud difraktsiooniprotsessi pöördumatus: valgus saab difrageeruda antud keskkondades ainult ühes suunas - madalamatelt difraktsioonijärkudelt kõrgematele. Arvutused näitavad, et kui ruumiliselt ja spektraalselt perioodilise võre saamiseks on kasutatud augu põletamise meetodit, siis esimese difraktsioonijärgu maksimaalne intensiivsus võib olla kuni $54 \%$, teisel järgul $30-40 \%$ pealelangeva impulsi intensiivsusest.

\section{R. SARAPUU}

\section{THE CAUSAL DIFFRACTION LATTICES IN SPACE-AND-TIME-DOMAIN HOLOGRAPHY}

It is shown that in diffraction processes in the media with spatial and spectral periodicities the causality principle plays an important role. Accordingly, the process of diffraction in this medium is irreversible: the light can diffract only in one direction from lower orders to the higher ones. It is shown that on using the hole-burning method to get the media with spatial and spectral periodicities, the first-order maximum diffraction efficiency may be $54 \%$ and that of the second-order $30-40 \%$ from the intensity of incident light. 